\title{
Nile red-dye based analysis of synthetic fibres for forensic applications.
}

\author{
DEVALLA, S., JOSEPH, O. and PRABHU, R.
}




\title{
Nile red dye-based analysis of microfibers for forensic applications
}

\author{
Sandhya Devalla ${ }^{1}$, Osheen Joseph ${ }^{2}$, Radhakrishna Prabhu ${ }^{3}$ \\ ${ }^{1}$ James Hutton Institute, Aberdeen, AB15 8QH, U.K. \\ ${ }^{2}$ International School of Photonics, Cochin University of Science \& Technology, Kochi, India \\ ${ }^{3}$ School of Engineering, Robert Gordon University, Aberdeen, United Kingdom
}

\begin{abstract}
Forensic evaluation of crime scenes normally involves examination of textile fibers, to find out the association between an individual and a crime scene, or between a suspect and a victim. The forensic samples normally include a mix of various types, sizes (micro to nano - scale) and shapes of natural and synthetic fibers, which are very difficult to differentiate/identify. Various sophisticated analytical instruments are being used to carry out the examination of these fibers. They involve various microscopy and spectroscopy based techniques, most of which are very complex and highly sensitive. Further, they may require a series of sample preparation steps to get high selectivity and are highly time consuming. Here we report a fluorescence microscopy based synthetic (plastic) fiber detection method using Nile Red (NR) dye, which provides high selectivity for synthetic fibers. The methodology involves the use of NR dye which selectively stains the fibers collected on filter papers following separation from samples/soils and water. The selectivity of NR towards the fibers is due to their non-polar property. Binding with NR makes the fibers fluoresce when viewed under a fluorescence microscope. This selectivity of NR for fibers makes the identification of fibers lot easier and less timeconsuming in forensic samples when compared to the more commonly used optical microscopy (where the presence of naturally-occurring substances of similar size can result in more errors). The paper will discuss optimisation of various parameters and method validation for detection of synthetic fibers and microplastics from soil samples. As an example, our method has shown to provide distinct clarity for the analysis of microfibers. The potential for the application of the method for faster forensics analysis will be discussed.
\end{abstract}

Keywords: Synthetic fibers, Forensics, Microplastic, Nile red, Fluorescence microscopy.

\section{INTRODUCTION}

Global production of plastics has reached over 300 million metric tons. The rate of production for plastics far exceed the rate of their degradation. As a result, billions of tons of plastic waste is being generated globally with massive accumulation of plastic wastes in land and water with no obvious strategy to tackle them. The plastics disposed in the water and land eventually breakdown to microplastics, widely accepted as particles $<5 \mathrm{~mm}$ size $\mathrm{e}^{1}$. The plastics that are manufactured in $5 \mathrm{~mm}$ size or less are called primary microplastics, eg: micro beads, microfibers from clothing, nurdles. There are plastics that breakdown into smaller sizes called secondary microplastics, example, from plastic waste such as bottles, plastic bags, disposable cutlery etc. An additional threat with microplastics has been that they act as vectors for other environmental contaminants such as PAH, PCB, pharmaceuticals thus enhancing their toxicities. Detection of microplastics in the environment is challenging. Currently, there is lack of standardized protocols for analysis of microplastics ${ }^{2}$. There is an urgent need to develop efficient methods for the detection and quantification of microplastics in order to understand the pathways of transport of microplastics in our environment to tackle this global scale problem. Methods of detection of microplastics should be robust, reliable and offer high through-put.

We have developed a method based on Nile Red (NR) dye staining of microplastics including microfibers and their detection by fluorescence microscopy. This method is robust, cheap and can be used as a fast screening method. The method could be particularly attractive in soil forensics with limited soil sample availability. In this paper we discuss the optimization of the NR dye method for microplastics. We will also explore the potential for application of this method to the area of soil forensics particularly microfiber examination. Soil forensics involves analysis of soil samples as trace evidence based on its characteristics ${ }^{3}$. The colour, pollen and even the particle size are some examples of the characteristics that forensic scientists look at in the soils. Both natural and synthetic fibers present in soils can also provide key evidence in criminal cases. Forensic fiber examination has been a fundamental part of criminal investigations for over 50 years ${ }^{4}$. 
Information on transferred fibers provide circumstantial evidence which help the forensic scientists to solve cases. The most frequent use is to provide evidence of possible contact between persons in crimes like murder, rape and sexual assault or aggravated assault. Then there is contact between person and other surfaces such as clothes, furniture, curtains, car seats etc., which contribute to cases such as armed robbery and terrorism.

NR dye (9-diethylamino-5- benzo[a]phenoxazinone (Figure 1) used in this study for tagging the microplastics and microfibers is selective for staining lipophilic and hydrophobic compounds. This dye is solvatochromatic which means its fluorescence intensity and colour depend upon the relative hydrophobicity of the solvent. For example, in the presence of polar solvents (e.g. dichloromethane) fluorescence is reddish while in less polar solvents (e.g. hexane) it is yellow-gold (Figure 2). On the other hand, the dye's fluorescence is quenched in aqueous environments thus acting as a hydrophobic probe. The solvatochromatic property of NR is due to its structure. NR has a phenoxazine structure which is uncharged, heterocyclic and rigid. There is a diethylamino end group attached to the rigid phenoxazine structure of NR. Molecules such as NR undergo twisted intramolecular charge transfer (TICT) the rate of which increases with increase in polarity of the medium. In the excited states, TICT molecules such as NR undergo an intramolecular electron transfer from the flexible diethylamino group towards the rigid phenoxazine molecule (acceptor). This is accompanied by a twist in the bond between the donor and the acceptor resulting in the molecule going from a planar in non-polar medium to a twisted state (where the donor and acceptor moieties are perpendicular to each other) in a polar medium. The photophysical process in NR is depicted in Figure 3. NR dye has therefore been used traditionally for the selective staining of lipids which are hydrophobic and nonpolar $^{5}$. As synthetic polymers share similar hydrophobic structure as that of lipids, NR has also been used successfully for the selective staining of microplastics and fibers. Maes et $\mathrm{al}^{6}$ were among the first to utilise the properties of NR for this purpose.<smiles>CCN(CC)c1ccc2nc3c4ccccc4c(=O)cc-3oc2c1</smiles>

Figure 1: Structure of Nile Red

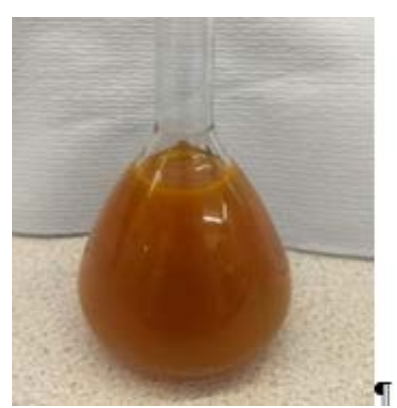

a

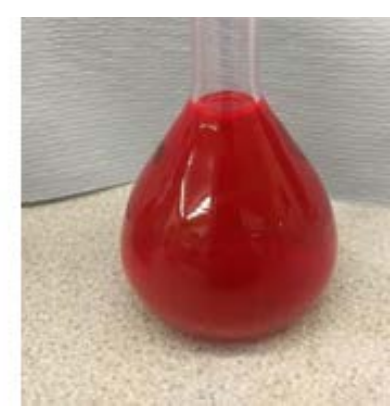

b

Figure 2: Stock solution of NR in (a) hexane and (b) dichloromethane 


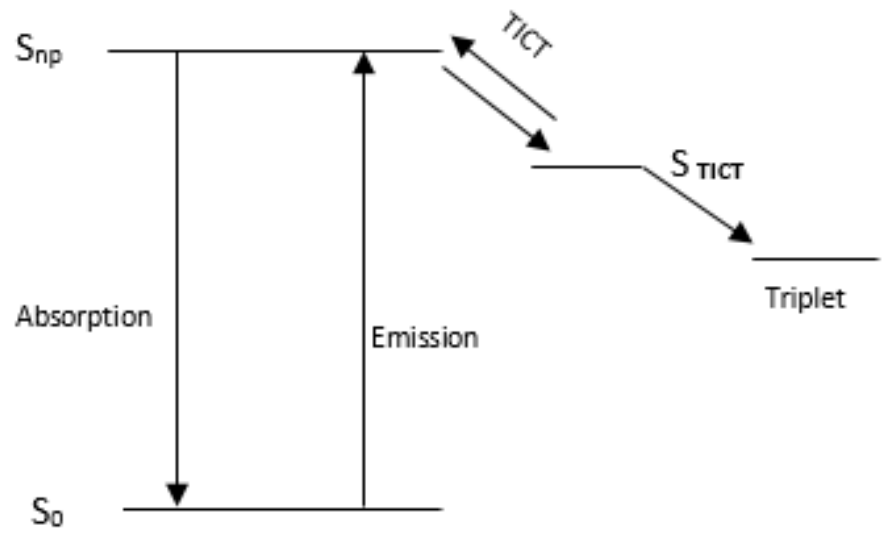

Figure 3: Photophysical processes in Nile Red where $\mathrm{S}_{\mathrm{np}}$ and $\mathrm{S}_{\text {TICT }}$ denote nonpolar and TICT excited states.

In this paper we present the new method where NR was used for the tagging of microplastics and detection using fluorescence microscope. NR dye offers the advantage of selective staining of microplastic polymers which fluoresce when illuminated with light at a certain wavelength and can be quantified by counting. Depending on the polymer polarity, a shift is expected in the fluorescence wavelength of NR when it is bonded to the microplastics. The fluorescence spectrum of NR dye in a non-polar solvent is shown in Figure 4 where the fluorescence maxima is at $600 \mathrm{~nm}$. This maximum is expected to shift depending on the polarity of the medium. Fluorescence based detection is an efficient method to detect microplastics due to its enhanced selectivity and sensitivity compared to the more routinely used optical microscopic methods. The method also has the potential to be applied directly to soil samples of small sizes to detect microplastics which is an advantage for forensic applications. This avoids the need for the separation procedure (described under methodology) altogether thus offering a fast screening method for soils.

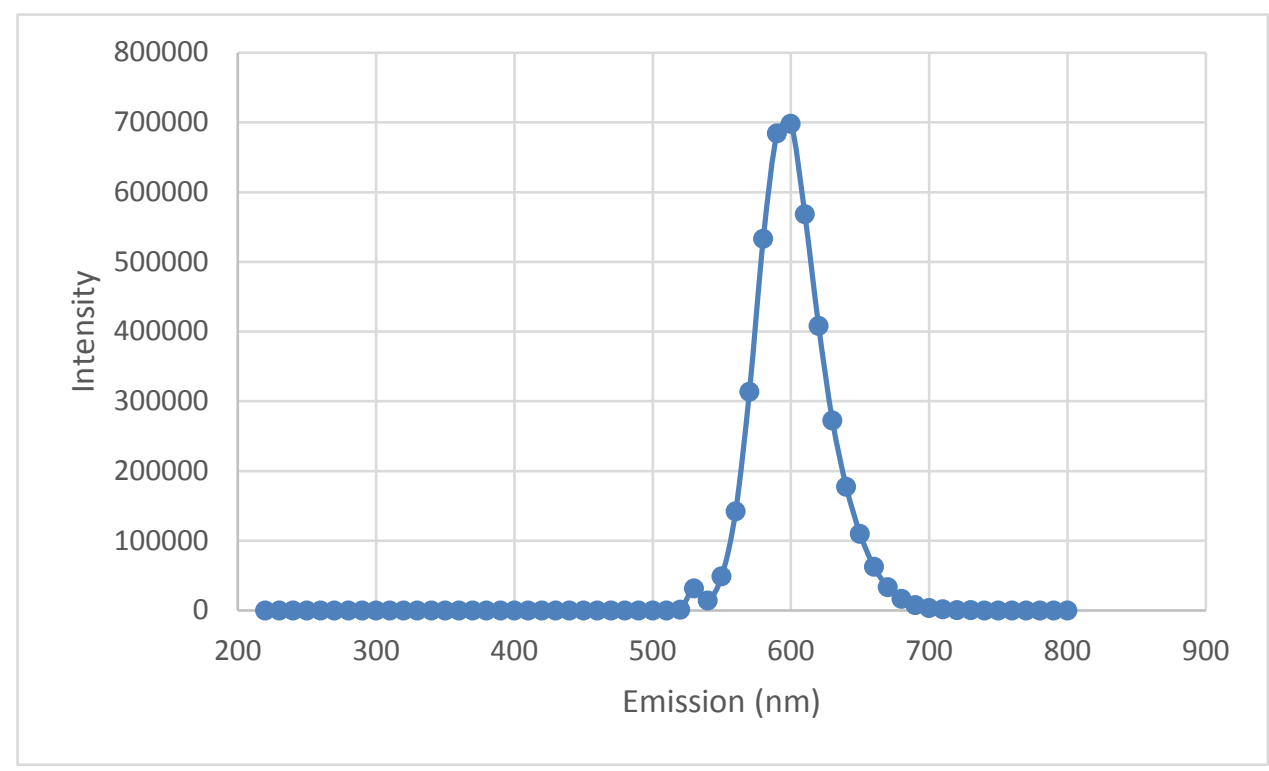

Figure 4: Fluorescence emission spectrum of Nile Red showing a maximum at $600 \mathrm{~nm}$ in hexane

\section{METHODOLOGY}

Prior to detection and quantification of microplastics by NR tagging and fluorescence microscopy, they have to firstly be separated from soils/sediments and collected onto a filter paper. The most widely used method to separate 
synthetic microfibers and microplastics from soils/sediments is by density separation. A highly dense salt solution such as zinc bromide (our laboratory method), is used to separate the microplastics from soils/sediments which have a lower density to that of the salt solution. Following density separation, the microplastics (fibers and fragments) floating on the salt solution are collected onto a Whatman filter paper, $55 \mathrm{~mm}$ dia. of $1.2 \mu \mathrm{m}$ particle size retention by filtration. The microplastics collected on the filter paper, are usually detected using an optical microscope and characterized based on their colour, shape, etc. The more commonly used detection which is by optical microscopy often leads to miscounting and misinterpretation of the microplastic polymers due to the presence of numerous other organic and inorganic components which also get collected on the filter paper. Thus, distinguishing between microplastics and other nonpolymer components can often be subjective. For example, with fibers, which are commonly encountered in soil samples, distinction between natural and synthetic can be challenging ${ }^{7}$. Also, it is difficult to identify between white and transparent fibers by optical microscopy. To overcome this drawback of optical microscopy, further confirmation is commonly carried out by Raman or FT-IR analysis. Although the spectroscopic techniques are useful to identify the type of microplastics, their analysis are expensive and time consuming.

\subsection{Preparation of Nile Red Dye and Staining of Microplastics}

NR stock solution of $100 \mathrm{mg} / \mathrm{l}$ was prepared in n-hexane. While preparing the NR stock solution in hexane, it was first dissolved in small amounts of acetone and then made up to a known volume in hexane. This is due to the lower solubility of NR in hexane. Working solutions of $0.5 \mathrm{mg} / \mathrm{l}$ and $2 \mathrm{mg} / \mathrm{l}$ were prepared from the stock solution after suitable dilutions.

The microplastics of different polymer types were prepared from everyday products which were initially cut into small pieces. These small plastic pieces were cryo-milled into microplastics using the SPEX Sample Prep - 6775 Polypropylene (PP), Polyvinylchloride (PVC), polytetrafluoroethylene (PTFE), Polycarbonate (PC), polyethylene (PE) from biodegradable bags and polyethylene terephthalate (PET) from a bottle. The microplastics were analysed by FTIR for confirming the type of the polymer. Filter papers (Whatman cellulose, ø-55 mm, pore size: $1.2 \mu \mathrm{m}$ ) containing different types of microplastics were stained with $200 \mu \mathrm{l}$ of the working NR solution. The filter papers were further carefully washed with $500 \mu \mathrm{l}$ water ( $50 \mu \mathrm{l}$ at a time) after about 30 mins of staining to remove excess dye. Fluorescence measurements of the filter papers were carried out using a Nikon SMZ1500 Stereoscopic Zoom Microscope and Leica DM5000 B Fluorescence Microscope. The samples were examined at a magnification of 10 and under three wavelengths: UV excitation $(360 / 40 \mathrm{~nm})$ - blue emission $(470 / 40 \mathrm{~nm})$, blue excitation $(480 / 40 \mathrm{~nm})$ - green emission $(530 / 27 \mathrm{~nm})$ and green excitation $(560 / 15 \mathrm{~nm})$ - red emission $(590 \mathrm{~nm})$. This experiment was carried out to determine if all the different types of microplastics showed fluorescence at specific wavelengths. A series of blank experiments were also performed to ensure there was no contamination or interference from material or reagents used.

\subsection{Application of the Nile Red Fluorescence to Micro Fibers}

Acrylic, coconut and barley root fibers were used in this study to determine the effectiveness of the method to distinguish between synthetic and natural fibers which is difficult by optical microscopy. Acrylic fibers were collected from a knitting yarn and cut into smaller pieces to obtain fibers of varied size range rather than a single standard size. These fibers were confirmed to be acrylic by FT-IR analysis. Natural fibers, namely coconut and barley root were washed and cut into smaller pieces

\section{RESULTS AND DISCUSSION}

\subsection{Fluorescence imaging of microplastics}

Microplastics fluoresced in all the three wavelength filters available for the fluorescence microscope- UV excitation $(360 / 40 \mathrm{~nm})$ - blue emission $(470 / 40 \mathrm{~nm})$, blue excitation $(480 / 40 \mathrm{~nm})$ - green emission $(530 / 27 \mathrm{~nm})$ and green excitation $(560 / 15 \mathrm{~nm})$ - red emission $(590 \mathrm{~nm})$. However, fluorescence was best in the green emission (Figures 5, 6) and hence this wavelength emission was chosen for subsequent analysis. The Figure shows images of the different microplastic polymer types. In the blue emission, not only plastics, but other organic matter also fluoresced for real environmental samples. In the red region, the background image appeared red. Although washing with water following NR dye staining was useful in reducing the background colour, in the red emission, often this was a problem. 
Results from blank experiments indicated no contamination. Maes ${ }^{6}$ et. al. noted that use of low quality plasticware such as pipette tips can introduce procedural contamination. It is therefore important to take all necessary precautions to minimise microplastic contamination in the laboratory. The different microplastics polymer types were also directly tested in the green emission before addition of NR dye to determine any autofluorescence. Amongst the different polymer types, biodegradable bag exhibited some fluorescence even without staining with NR dye. This could possibly be due to the various additives and pigments that could have been added during the manufacturing process.

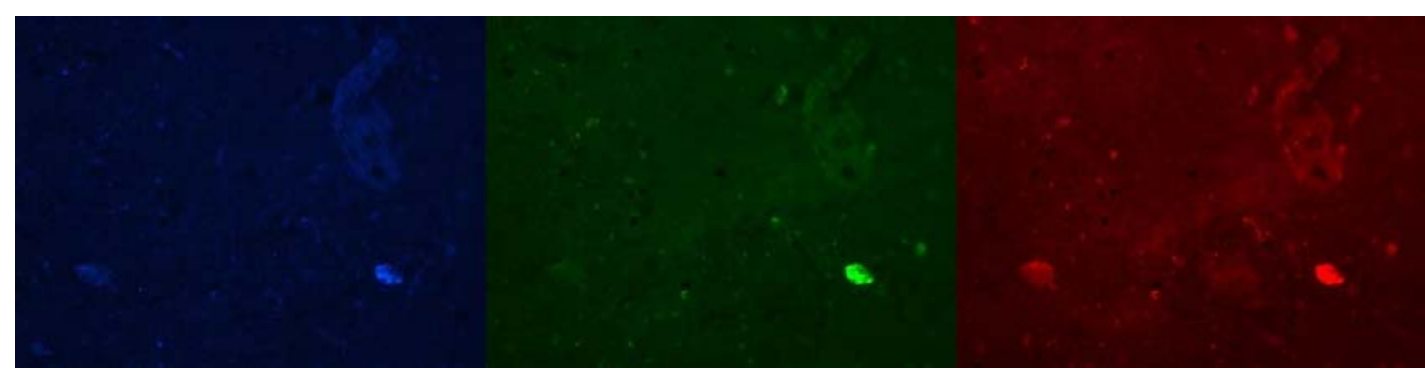

Figure 5: Example image of a microplastic particle detected upon Nile Red staining at all three wavelengths.

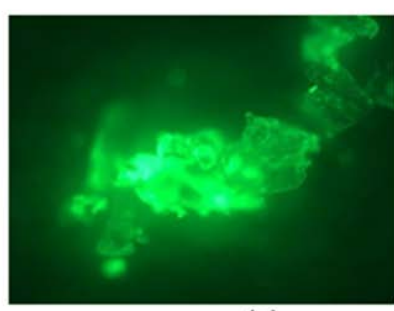

(a)

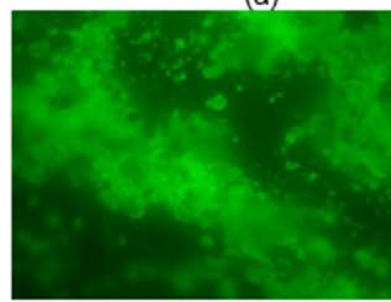

(d)

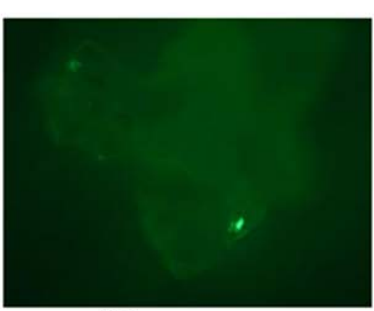

(b)

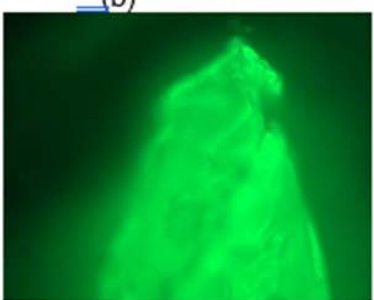

(e)

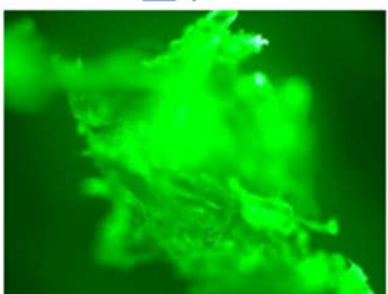

(g)

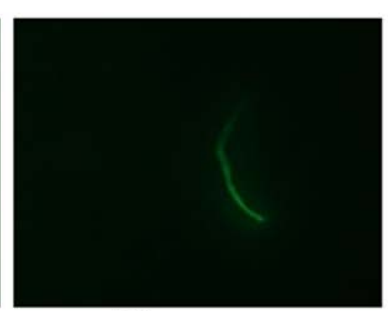

(c)

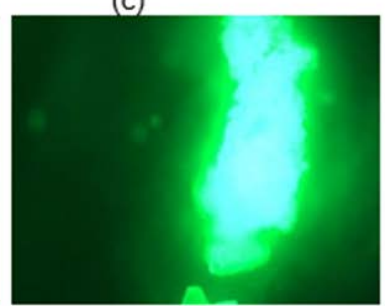

(f)

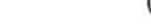

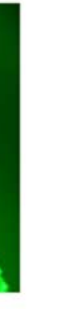

Figure 6: Images demonstrating the fluorescence of (a) PVC, (b) PC, (c) PTFE, (d) PP, (e) PET, (f) biodegradable bag, (g) PE upon Nile Red staining.

\subsection{Application of Nile Red Fluorescence to Micro Fibers}

Natural fibers (coconut, barley root) fluoresced even without addition of NR. However, there was clear distinction between natural and synthetic fibers (Figure 7). The inner part of natural fibers was not homogenous, and their shape was non-uniform across their length compared to that of synthetic fibers which were more uniform throughout. This will be useful for forensics applications where distinguishing between natural and synthetic fibers is a useful parameter in elucidating important information. For future work, animal fibers could also be tested. Distinguishing between natural 
plant and animal fibers is likely to be possible due to the inherent polymer differences between them. While plant fibers contain cellulose polymers, animal fibers are usually protein based. More systematic studies and building of large datasets are however required in this area.

With further optimization, the method of NR staining can also be useful for direct application to forensic soil samples where only limited sample sizes are available. In future, the solvatochromatic property of NR could be utilized for the construction of fluorescence probes to enable in-situ identification in forensic samples. Based on the polarity of the polymer types, their bonding with NR is expected to shift the fluorescence maxima. Thus, it is envisaged that fluorescence probes constructed with narrow wavelength bandwidths can differentiate between the different polymer types. The construction of such probes would offer cheaper microplastic quantification method where polymer types can also be analysed. Additionally, direct application on samples can reduce microplastic and fiber losses instead of samples having to be transported from the crime site to the laboratory for analysis.

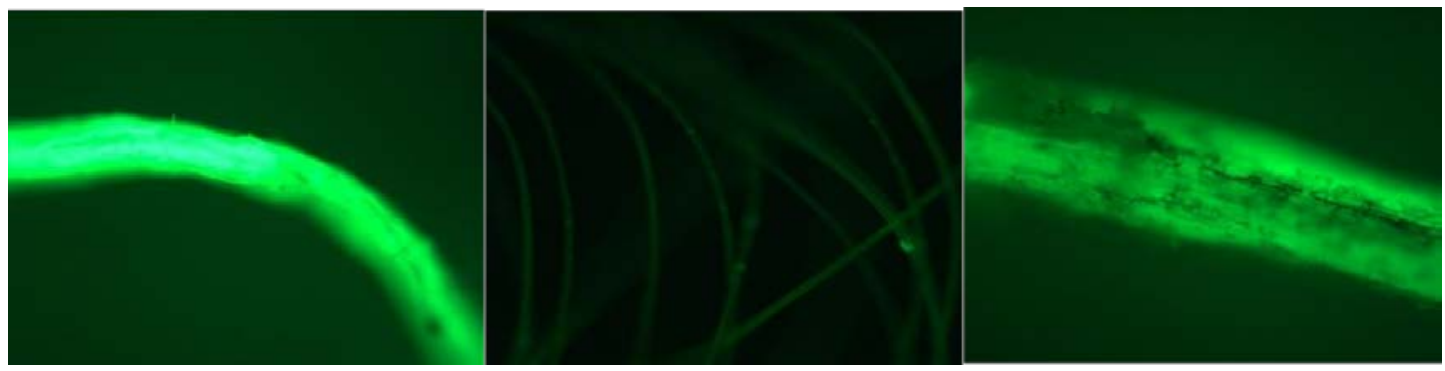

Figure 7: Fluorescence of (a) barley root, (b) acrylic and (c) coconut fiber upon Nile Red staining

\section{CONCLUSION}

The findings from this study show that staining with NR dye followed by fluorescence microscope detection is an efficient method for the detection of microplastics and microfibers. It was possible to distinguish between natural and synthetic fibers. Fluorescence measurements were carried out in green emission wavelength for increased fluorescence and reducing interferences from naturally fluorescing organic matter. However, for future work, the shift in absorption and fluorescence peak of NR when bonded to polymers can be noted and an excitation and emission filter of that specific bandwidth can be designed. This would help to excite the dye molecule that is only bonded to polymers and thus not only reduce background noise, but has the potential to distinguish between polymer types. Automated quantification techniques can be explored in future for counting of microplastics along with photo-documentation. The present research provides fundamental observations which can be further developed for the effective quantification of microplastics and microfibers for forensics applications. Undoubtedly, an emerging field is taking shape.

\section{Acknowledgements}

This work was supported by the Rural \& Environment Science \& Analytical Services Division of the Scottish Government and the GCRF funding from the Scottish Funding Council.

\section{REFERENCES}

[1] Hidalgo-Ruz, V., Gutow, L., Thompson R.C., Thiel, M., "Microplastics in the Marine Environment: A Review of the Methods Used for Identification and Quantification”. Environ Sci Technol. 46(6), 3060-75 (2012).

[2] Mai, L., Bao, L.J., Shi, L., Wong, C.S., Zeng, E.Y., “A review of methods for measuring microplastics in aquatic environments. Environ Sci Pollut Res Int., 25(12), 11319-11332 (2018).

[3] Carpi, A, Mital, J., “The Expanding Use of Forensics in Environmental Science”. Environ Sci Technol.; 34(11):254A-261A (2000).

[4] Singh, J.P., Singh K., "Fiber Examination in Forensic Science”. J Textile Sci Eng (2013) 3:e116. doi: 10.4172/2165-8064.1000e116. 
[5] Cooksey, K.E., Guckert, J.B., Williams, S.A., Callis, P.R., "Fluorometric determination of the neutral lipid content of microalgal cells using Nile Red”. Journal of microbiological methods, 6(6):333-345 (1987).

[6] Maes, T., Jessop, R., Wellner, N., Haupt K, Mayes A.G., “A rapid-screening approach to detect and quantify microplastics based on fluorescent tagging with Nile Red”. Sci Rep., 16;7(1):44501 (2017).

[7] Zubris, K.A V., Richards B.K., "Synthetic fibers as an indicator of land application of sludge”. Environ Pollut:;138(2):201-11 (2005). 